\title{
Graphitic Carbon Nitride Photocatalysts for Degradation of Organic Pollutants
}

\author{
Huimin Li ${ }^{\text {a }}$, Huixian Zhang ${ }^{b}$, Keming Fang ${ }^{c}$, Lining Yang ${ }^{d}$, Jianrong Chen ${ }^{e^{*}}$ \\ College of Geography and Environmental Sciences, Zhejiang Normal University, Jinhua, 321004, China. \\ alihimin2008@qq.com, ${ }^{b} 953878987 @ q q . c o m,{ }^{c}$ sky65@zjnu.cn, dyln@zjnu.cn, ${ }^{\mathrm{a}}$ cjr@zjnu.cn \\ *Corresponding author: Tel: +86 579 82291275; Fax: +86 57982282273
}

Keywords: Graphitic carbon nitride, Photodegradation, Organic pollutants.

\begin{abstract}
Organic pollutants have posed threat to human health and other creature. Photocatalytic technology arises, because it can use solar energy to degrade pollutants. Hence, graphitic carbon nitride $\left(\mathrm{g}-\mathrm{C}_{3} \mathrm{~N}_{4}\right)$ with excellent photocatalytic performance has attracted global attention. This paper presents the introduction of $g-\mathrm{C}_{3} \mathrm{~N}_{4}$ and recent achievements of $g-\mathrm{C}_{3} \mathrm{~N}_{4}$ composites in the photodegradation of organic pollutants.
\end{abstract}

\section{Introduction}

Graphitic carbon nitride ( $\left(\mathrm{g}-\mathrm{C}_{3} \mathrm{~N}_{4}\right)$ as one of the representative and promising photocatalysts, which is composed of two earth-abundant elements: carbon and nitrogen, has become a very hot research topic due to its excellent photocatalysis properity with the bandgap $2.7 \mathrm{eV}$, outstanding chemical stability, fascinating optical and electrical properties[1]. These unique characteristics make g- ${ }_{3} \mathrm{~N}_{4}$ be widely applied in various photocatalytic fields, such as pollutant degradation[2], hydrogen production from water splitting[3], $\mathrm{CO}_{2}$ reduction[4], and bacterial disinfection[4].

Many strategies have been reported for the synthesis of $\mathrm{g}-\mathrm{C}_{3} \mathrm{~N}_{4}$, and different methods could prepare different materials with specific topography and electronic properties. Such methods include solvothermal[5,6], thermal polymerization[7], electrochemical deposition[8], solid state[9], etc. Thermal polymerization has been the most commonly used synthetic method owing to its simple operation steps and short synthetic cycle. The precursors derive from a verity of carbon/nitrogen-rich molecules, such as urea, melamine, cyanate, thiourea, cyanamide, thiocyanate, guanidine hydrochloride and so on. In a typical synthesis, melamine was put into an alumina crucible which was heated to $550{ }^{\circ} \mathrm{C}$ in a muffle furnace with a heating rate of $2{ }^{\circ} \mathrm{C} / \mathrm{min}$. After calcination for $2 \mathrm{~h}$, the alumina crucible was cooled naturally to room temperature. The sample was collected and ground into powder[10].

\section{Applications of $g-C_{3} N_{4}$ in the photodegradation of organic pollutants}

Nowadays, people are faced with environment problem, which promotes the development of semiconductor photocatalysis. $\mathrm{G}-\mathrm{C}_{3} \mathrm{~N}_{4}$ is one of non-mental photocatalysts, and has been widely used to photodegrade a variety of organic pollutants, including rhodamine $\mathrm{B}(\mathrm{RhB})$, methylene blue (MB), methyl orange (MO) and so on[11]. Table 1 shows the invention of a wide variety of g- $\mathrm{C}_{3} \mathrm{~N}_{4}$ nanocomposites for the photodegradation of organic pollutants. Additionally, their photocatalytic performance is also displayed in the table. It is well known that photogenerated electrons $\left(\mathrm{e}^{-}\right)$and holes $\left(\mathrm{h}^{+}\right)$could generate radials, such as hydroxyl radicals $(\bullet \mathrm{OH})$ and superoxide anion radicals $\left(\cdot \mathrm{O}_{2}{ }^{-}\right)$which have strong redox property[12]. Once reactive species are produced, they will oxidize organic pollutants during photocatalytic reactions. 
Table 1 Recent achievements of g- $\mathrm{C}_{3} \mathrm{~N}_{4}$ composites in the photodegradation of pollutants.

\begin{tabular}{|c|c|c|c|c|c|}
\hline Catalyst & Pollutant & $\begin{array}{c}\text { Catalyst } \\
\text { dose } \\
(\mathrm{g} / \mathrm{L})\end{array}$ & $\begin{array}{c}\text { Pollutant } \\
\text { concentration }\end{array}$ & $\begin{array}{c}\text { Degradation } \\
\text { efficiency }\end{array}$ & Reference \\
\hline $\mathrm{BiOBr} / \mathrm{g}-\mathrm{C}_{3} \mathrm{~N}_{4}$ & RhB & 0.5 & $20 \mathrm{mg} / \mathrm{L}$ & $100 \%$ in $40 \mathrm{~min}$ & [13] \\
\hline $\mathrm{Bi}_{2} \mathrm{O}_{3} / g-\mathrm{C}_{3} \mathrm{~N}_{4}$ & RhB & 1.0 & $1.0 \times 10^{-5} \mathrm{M}$ & $95 \%$ in $120 \mathrm{~min}$ & [14] \\
\hline $\mathrm{In}_{2} \mathrm{VO}_{4} / \mathrm{g}-\mathrm{C}_{3} \mathrm{~N}_{4}$ & $\mathrm{RhB}$ & 0.3 & $0.002 \mathrm{M}$ & $100 \%$ in $20 \mathrm{~min}$ & [15] \\
\hline $\mathrm{NaTaO}_{3} / \mathrm{g}-\mathrm{C}_{3} \mathrm{~N}_{4}$ & RhB & 0.25 & $5 \mathrm{mg} / \mathrm{L}$ & $100 \%$ in $90 \mathrm{~min}$ & [16] \\
\hline $\mathrm{SnO}_{2} / g-\mathrm{C}_{3} \mathrm{~N}_{4}$ & $\mathrm{RhB}$ & 2.0 & $10 \mathrm{mg} / \mathrm{L}$ & $100 \%$ in $120 \mathrm{~min}$ & {$[17]$} \\
\hline $\mathrm{ZnO} / \mathrm{g}-\mathrm{C}_{3} \mathrm{~N}_{4}$ & $\mathrm{MO}$ & 3.0 & $5 \mathrm{mg} / \mathrm{L}$ & $95 \%$ in $100 \mathrm{~min}$ & [18] \\
\hline $\mathrm{BiOCl} / \mathrm{g}-\mathrm{C}_{3} \mathrm{~N}_{4}$ & $\mathrm{MO}$ & 1.0 & $10 \mathrm{mg} / \mathrm{L}$ & $100 \%$ in $80 \mathrm{~min}$ & [19] \\
\hline $\mathrm{Ag}_{3} \mathrm{PO}_{4} / \mathrm{g}-\mathrm{C}_{3} \mathrm{~N}_{4}$ & $\mathrm{MO}$ & 0.25 & $10 \mathrm{mg} / \mathrm{L}$ & $100 \%$ in $20 \mathrm{~min}$ & {$[20]$} \\
\hline $\mathrm{ZnFe}_{2} \mathrm{O}_{4} / g-\mathrm{C}_{3} \mathrm{~N}_{4}$ & $\mathrm{MO}$ & 0.25 & $10 \mathrm{mg} / \mathrm{L}$ & $100 \%$ in $180 \mathrm{~min}$ & [21] \\
\hline $\mathrm{Co}_{3} \mathrm{O}_{4} / \mathrm{g}-\mathrm{C}_{3} \mathrm{~N}_{4}$ & $\mathrm{MO}$ & 1.0 & $10 \mathrm{mg} / \mathrm{L}$ & $100 \%$ in $180 \mathrm{~min}$ & [22] \\
\hline $\mathrm{MoO}_{3} / \mathrm{g}-\mathrm{C}_{3} \mathrm{~N}_{4}$ & $\mathrm{MB}$ & 1.0 & $10 \mathrm{mg} / \mathrm{L}$ & $95 \%$ in $180 \mathrm{~min}$ & [23] \\
\hline $\mathrm{Zn}_{0.8} \mathrm{Cd}_{0.2} \mathrm{~S} / \mathrm{g}-\mathrm{C}_{3} \mathrm{~N}_{4}$ & $\mathrm{MB}$ & 1.0 & $10 \mathrm{mg} / \mathrm{L}$ & $100 \%$ in $180 \mathrm{~min}$ & [24] \\
\hline $\mathrm{Ag}_{3} \mathrm{VO}_{4} / \mathrm{g}-\mathrm{C}_{3} \mathrm{~N}_{4}$ & $\mathrm{MB}$ & 0.5 & $10 \mathrm{mg} / \mathrm{L}$ & $95 \%$ in $30 \mathrm{~min}$ & [25] \\
\hline N-doped $\mathrm{TiO}_{2} / \mathrm{g}-\mathrm{C}_{3} \mathrm{~N}_{4}$ & MB & 1.0 & $10 \mathrm{mg} / \mathrm{L}$ & $100 \%$ in $60 \mathrm{~min}$ & {$[26]$} \\
\hline$\alpha-\mathrm{Fe}_{2} \mathrm{O}_{3} / g-\mathrm{C}_{3} \mathrm{~N}_{4}$ & Directred81 & 1.0 & $5.0 \times 10^{-5} \mathrm{M}$ & $70 \%$ in $120 \mathrm{~min}$ & [27] \\
\hline $\mathrm{BiOCl}_{\mathrm{x}} \mathrm{Br}_{1-\mathrm{x}} / \mathrm{g}-\mathrm{C}_{3} \mathrm{~N}_{4}$ & Rhodamine 640 & 0.25 & $9 \mathrm{mg} / \mathrm{L}$ & $55 \%$ in $90 \mathrm{~min}$ & {$[28]$} \\
\hline $\mathrm{Ag} / \mathrm{AgVO}_{3} / \mathrm{g}-\mathrm{C}_{3} \mathrm{~N}_{4}$ & Basic fuchsin & 1.0 & $20 \mathrm{mg} / \mathrm{L}$ & $99.3 \%$ in $70 \mathrm{~min}$ & [29] \\
\hline $\mathrm{WO}_{3} / \mathrm{g}-\mathrm{C}_{3} \mathrm{~N}_{4}$ & Basic fuchsin & 2.0 & $1.0 \times 10^{-5} \mathrm{M}$ & $75 \%$ in $60 \mathrm{~min}$ & [30] \\
\hline $\mathrm{Cu}_{2} \mathrm{O} / \mathrm{g}-\mathrm{C}_{3} \mathrm{~N}_{4}$ & Acid Orange-II & 0.1 & $40 \mathrm{mg} / \mathrm{L}$ & $95 \%$ in $180 \mathrm{~min}$ & [31] \\
\hline $\mathrm{g}-\mathrm{C}_{3} \mathrm{~N}_{4} / \mathrm{CdS} / \mathrm{RGO}$ & Congo red & 0.4 & $20 \mathrm{mg} / \mathrm{L}$ & $100 \%$ in $20 \mathrm{~min}$ & [32] \\
\hline $\mathrm{CuFe}_{2} \mathrm{O}_{4} / \mathrm{g}-\mathrm{C}_{3} \mathrm{~N}_{4}$ & Orange II & 0.1 & $0.028 \mathrm{mM}$ & $90 \%$ in $180 \mathrm{~min}$ & [33] \\
\hline $\mathrm{P}-\mathrm{g}-\mathrm{C}_{3} \mathrm{~N}_{4} / \mathrm{ZnLn}_{2} \mathrm{~S}_{4}$ & 4-nitroaniline & 1.0 & $10 \mathrm{mg} / \mathrm{L}$ & $99.4 \%$ in $90 \mathrm{~min}$ & [34] \\
\hline$g-\mathrm{C}_{3} \mathrm{~N}_{4} / \mathrm{TiO}_{2}(\mathrm{TCN})$ & ciprofloxacin & 0.5 & $20 \mathrm{mg} / \mathrm{L}$ & $97.3 \%$ in $150 \mathrm{~min}$ & [35] \\
\hline $\mathrm{g}-\mathrm{C}_{3} \mathrm{~N}_{4} / \mathrm{WO}_{3}$ & acetaldehyde & 0.1 & $11.2 \mu \mathrm{mol} / \mathrm{L}$ & $100 \%$ in $24 \mathrm{~h}$ & [35] \\
\hline mpg- $\mathrm{C}_{3} \mathrm{~N}_{4} / \mathrm{Co}_{3} \mathrm{O}_{4}$ & bisphenol & 0.4 & $15 \mathrm{mg} / \mathrm{L}$ & $93.6 \%$ in $180 \mathrm{~min}$ & [36] \\
\hline $\mathrm{g}-\mathrm{C}_{3} \mathrm{~N}_{4} / \mathrm{ZnIn}_{2} \mathrm{~S}_{4}$ & phenol & 0.4 & $10 \mathrm{mg} / \mathrm{L}$ & $73.2 \%$ in $4 \mathrm{~h}$ & [37] \\
\hline $\mathrm{Bi}_{5} \mathrm{Nb}_{3} \mathrm{O}_{15} / g-\mathrm{C}_{3} \mathrm{~N}_{4}$ & 4-chlorophenol & 1.0 & $10 \mathrm{mg} / \mathrm{L}$ & $100 \%$ in $60 \mathrm{~min}$ & [38] \\
\hline $\mathrm{Pd} / \mathrm{mpg}-\mathrm{C}_{3} \mathrm{~N}_{4}$ & bisphenol A & 0.5 & $20 \mathrm{mg} / \mathrm{L}$ & $93.9 \%$ in $180 \mathrm{~min}$ & [39] \\
\hline $\mathrm{Ag} / \mathrm{AgBr} / \mathrm{g}-\mathrm{C}_{3} \mathrm{~N}_{4}$ & 2-chlorophenol & $1 . .0$ & $20 \mathrm{mg} / \mathrm{L}$ & $70.51 \%$ in $4 \mathrm{~h}$ & [40] \\
\hline
\end{tabular}

\section{Summary}

In the view of the scientific reports, great efforts have been devoted to prepare $g-\mathrm{C}_{3} \mathrm{~N}_{4}$ composites. It is delightful that remarkable achievements have been made on the field of photodegradation of pollutants. However, the mechanism of photocatalysis is not clear. More importantly, these materials still have long way to achieve practical application.

\section{Acknowledgment}

This research was supported by National Natural Science Foundation of China (No. 21275131).

\section{References}

[1] Cao, S., Low J., Yu J., Jaroniec, M. (2015) Polymeric Photocatalysts based on Graphitic Carbon Nitride. Advanced materials, 27, 2150-2176.

[2] Yan, S.C., Li, Z.S., Zou, Z.G. (2010) Photodegradation of Rhodamine B and Methyl Orange over Boron-doped G- $\mathrm{C}_{3} \mathrm{~N}_{4}$ under Visible Llight Irradiation. Langmuir the Acs Journal of Surfaces \& Colloids, 26, 3894-3901.

[3] Kohl, S.W., Weiner, L., Schwartsburd, L. (2009) Consecutive Thermal $\mathrm{H}_{2}$ and Llight-induced $\mathrm{O}_{2}$ Evolution from Water Promoted by A Metal Complex. Science, 324, 74-77. 
[4] Roy, S.C., Varghese, O.K., Paulose, M. (2010) Toward Solar Fuels: Photocatalytic Conversion of Carbon Dioxide to Hydrocarbons. Acs Nano, 4, 1259-1278.

[5] Guo, Q., Xie, Y., Wang, X. (2003) Characterization of Well-crystallized Graphitic Carbon Nitride Nanocrystallites via A Benzene-thermal Route at Low Temperatures. Chemical Physics Letters, 380, 84-87.

[6] Cui, Y., Ding, Z., Fu, X. (2012) Construction of Conjugated Carbon Nitride Nanoarchitectures in Solution at Low Temperatures for Photoredox Catalysis. Angewandte Chemie, 124, 11814-11818.

[7] Groenewolt, M., Antonietti, M. (2005) Synthesis of G- $\mathrm{C}_{3} \mathrm{~N}_{4}$ Nanoparticles in Mesoporous Silica Host Matrices, 17, 1789-1792.

[8] Lv, Q., Cao, C., Zhang, Y. (2004) The Preparation of Insoluble Fibroin Films Induced by Degummed Fibroin or Fibroin Microspheres. Journal of Materials Science Materials in Medicine, 15, 1193-1197.

[9] Fu, M., Pi, J., Dong, F. (2013) A Cost-effective Solid-state Approach to Synthesize G-C ${ }_{3} \mathrm{~N}_{4}$ Coated $\mathrm{TiO}_{2}$ Nanocomposites with Enhanced Visible Light Photocatalytic Activity. International Journal of Photoenergy, 2013, 10196-10202.

[10] Zhang, X., Xie, X., Wang, H. (2013) ChemInform Abstract: Enhanced Photo Responsive Ultrathin Graphitic-phase $\mathrm{C}_{3} \mathrm{~N}_{4}$ Nanosheets for Bioimaging. Cheminform, 135, 18.

[11] Mamba, G., Mishra, A.K. (2016) Graphitic Carbon Nitride $\left(\mathrm{g}^{\left.-\mathrm{C}_{3} \mathrm{~N}_{4}\right)}\right.$ Nanocomposites: A New and Exciting Generation of Visible Light Driven Photocatalysts for Environmental Pollution Remediation. Applied Catalysis B: Environmental, 198, 347-377.

[12] Lam, S.M., Sin, J.C., Mohamed, A.R. (2016) A Review on Photocatalytic Application of G-C ${ }_{3} \mathrm{~N}_{4}$ /Semiconductor (CNS) Nanocomposites towards the Erasure of Dyeing Wastewater. Materials Science in Semiconductor Processing, 47, 62-84.

[13] Fu J., Tian, Y., Chang, B. (2012) BiOBr-Carbon Nitride Heterojunctions: Synthesis, Enhanced Activity and Photocatalytic Mechanism. Journal of Materials Chemistry, 22, 21159.

[14] Zhang, J., Hu, Y., Jiang, X. (2014) Design of A Direct Z-scheme Photocatalyst: Preparation and Characterization of $\mathrm{Bi}_{2} \mathrm{O}_{3} / g-\mathrm{C}_{3} \mathrm{~N}_{4}$ with High Visible Light Activity. J Hazard Mater, 280, 713-722.

[15] Shi, W., Guo, F., Chen, J. (2014) Hydrothermal Synthesis of $\mathrm{InVO}_{4} /$ Graphitic Carbon Nitride Heterojunctions and Excellent Visible-light-driven Photocatalytic Performance for Rhodamine B. Journal of Alloys and Compounds, 612, 143-148.

[16] Kumar, S., Kumar, B., Surendar, T. (2014) $\mathrm{G}-\mathrm{C}_{3} \mathrm{~N}_{4} / \mathrm{NaTaO}_{3}$ Organic-inorganic Hybrid Nanocomposite: High-performance and Recyclable Visible Light Driven Photocatalyst. Materials Research Bulletin, 49, 310-318.

[17] Zang, Y., Li, L., Li X. (2014) Synergistic Collaboration of G- $\mathrm{C}_{3} \mathrm{~N}_{4} / \mathrm{SnO}_{2}$ Composites for Enhanced Visible-light Photocatalytic Activity. Chemical Engineering Journal, 246, 277-286.

[18]Sun, J.X., Yuan, Y.P., Qiu, L.G. (2012) Fabrication of Composite Photocatalyst G- $\mathrm{C}_{3} \mathrm{~N}_{4}-\mathrm{ZnO}$ and Enhancement of Photocatalytic Activity under Visible Light. Dalton Transactions, 41, 6756.

[19] Wang, X.J., Wang, Q., Li, F.T. (2013) Novel BiOCl- $\mathrm{C}_{3} \mathrm{~N}_{4}$ Heterojunction Photocatalysts: In Situ Preparation via An Ionic-liquid-assisted Solvent-thermal Route and Their Visible-light Photocatalytic Activities. Chemical Engineering Journal, 234, 361-371.

[20] Kumar, S., Surendar, T., Baruah, A. (2013) Synthesis of A Novel and Stable G-C $\mathrm{N}_{4}-\mathrm{Ag}_{3} \mathrm{PO}_{4}$ Hybrid Nanocomposite Photocatalyst and Study of The Photocatalytic Activity under Visible Light Irradiation. Journal of Materials Chemistry A, 1, 5333.

[21] Zhang, S., Li, J., Zeng, M. (2013) In Situ Synthesis of Water-soluble Magnetic Graphitic 
Carbon Nitride Photocatalyst and Its Synergistic Catalytic Performance. ACS applied materials \& interfaces, 5, 12735-12743.

[22] Han, C., Ge, L., Chen, C. (2014) Novel Visible Light Induced $\mathrm{Co}_{3} \mathrm{O}_{4}-\mathrm{g}-\mathrm{C}_{3} \mathrm{~N}_{4}$ Heterojunction Photocatalysts for Efficient Degradation of Methyl Orange. Applied Catalysis B: Environmental, 147, 546-553.

[23] Huang, L., Xu, H., Zhang, R. (2013) Synthesis and Characterization of $\mathrm{G}_{-} \mathrm{C}_{3} \mathrm{~N}_{4} / \mathrm{MoO}_{3}$ Photocatalyst with Improved Visible-light Photoactivity. Applied Surface Science, 283, 25-32.

[24] Li, D., Wu, Z., Xing, C. Novel $\mathrm{Zn}_{0.8} \mathrm{Cd}_{0.2} \mathrm{~S} / \mathrm{g}-\mathrm{C}_{3} \mathrm{~N}_{4}$ Heterojunctions with Superior Visible-light Photocatalytic Activity: Hydrothermal Synthesis and Mechanism Study. Journal of Molecular Catalysis A Chemical, 395, 261-268.

[25] Zhu, T., Song, Y., Ji, H. (2015) Synthesis of G-C $\mathrm{N}_{4} / \mathrm{Ag}_{3} \mathrm{VO}_{4}$ Composites with Enhanced Photocatalytic Activity under Visible Light Iiation. Chemical Engineering Journal, 271, 96-105.

[26] Wang, X.J., Yang, W.Y., Li, F.T. (2013) In Situ Microwave-assisted Synthesis of Porous $\mathrm{N}-\mathrm{TiO}_{2} / \mathrm{g}-\mathrm{C}_{3} \mathrm{~N}_{4}$ Heterojunctions with Enhanced Visible-light Photocatalytic Properties. Industrial \& Engineering Chemistry Research, 52, 17140-17150.

[27] Theerthagiri, J., Senthil, R.A., Priya, A. (2014) Photocatalytic and Photoelectrochemical Studies of Visible-light Active $\alpha-\mathrm{Fe}_{2} \mathrm{O}_{3}-\mathrm{g}-\mathrm{C}_{3} \mathrm{~N}_{4}$ Nanocomposites. Rsc Advances, 4, 38222-38229.

[28] Shi, S., Gondal, M.A., Rashid, S.G. (2014) Synthesis of g- $\mathrm{C}_{3} \mathrm{~N}_{4} / \mathrm{BiOCl}_{\mathrm{x}} \mathrm{Br}_{1-\mathrm{x}} \mathrm{Hybrid}$ Photocatalysts and The Photoactivity Enhancement Driven by Visible Light. Colloids and Surfaces A: Physicochemical and Engineering Aspects, 461, 202-211.

[29] Zhao, W., Guo, Y., Wang, S. (2015) A Novel Ternary Plasmonic Photocatalyst: Ultrathin $\mathrm{G}_{-} \mathrm{C}_{3} \mathrm{~N}_{4}$ Nanosheet Hybrided by $\mathrm{Ag} / \mathrm{AgVO}_{3}$ Nanoribbons with Enhanced Visible-light Photocatalytic Performance. Applied Catalysis B: Environmental, 165, 335-343.

[30] Chen, S., Hu, Y., Jiang, X. (2015) Fabrication and Characterization of Novel Z-scheme Photocatalyst $\mathrm{WO}_{3} / \mathrm{g}-\mathrm{C}_{3} \mathrm{~N}_{4}$ with High Efficient Visible Light Photocatalytic Activity. Materials Chemistry and Physics, 149-150, 512-521.

[31] Peng, B., Zhang, S., Yang, S. (2014) Synthesis and Characterization of $\mathrm{G}-\mathrm{C}_{3} \mathrm{~N}_{4} / \mathrm{Cu}_{2} \mathrm{O}$ Composite Catalyst with Enhanced Photocatalytic Activity under Visible Light Irradiation. Materials Research Bulletin, 56, 19-24.

[32] Pawar, R.C., Khare, V., Lee, C.S. (2014) Hybrid Photocatalysts Using Graphitic Carbon Nitride/Cadmium Sulfide/Reduced Graphene Oxide $\left(\mathrm{g}-\mathrm{C}_{3} \mathrm{~N}_{4} / \mathrm{CdS} / \mathrm{RGO}\right)$ for Superior Photodegradation of Organic Pollutants under UV and Visible Light. Dalton Transactions, 43, 12514-12527.

[33] Yao, Y., Lu, F., Zhu, Y. (2015) Magnetic Core-shell $\mathrm{CuFe}_{2} \mathrm{O}_{4} @ \mathrm{C}_{3} \mathrm{~N}_{4}$ Hybrids for Visible Light Photocatalysis of Orange II. J Hazard Mater, 297, 224-233.

[34] Chen, W., Liu, T.Y., Huang, T. (2016) Novel Mesoporous P-doped Graphitic Carbon Nitride Nanosheets Coupled with $\mathrm{ZnIn}_{2} \mathrm{~S}_{4}$ Nanosheets as Efficient Visible Light Driven Heterostructures with Remarkably Enhanced Photo-reduction Activity. Nanoscale, 8, 3711-3719.

[35] Jiang, Z., Zhu, C., Wan, W. (2016) Constructing Graphite-like Carbon Nitride Modified Hierarchical Yolk-shell $\mathrm{TiO}_{2}$ Spheres for Water Pollution Treatment and Hydrogen Production. Journal of Materials Chemistry A, 4, 1806-1818.

[36] Qiu, P., Chen, H., Jiang, F. (2014) Cobalt Modified Mesoporous Graphitic Carbon Nitride with Enhanced Visible-light Photocatalytic Activity. RSC Adv, 4, 39969-39977.

[37] Liu, H., Jin, Z., Xu Z. Fabrication of $\mathrm{ZnIn}_{2} \mathrm{~S}_{4}-\mathrm{g}-\mathrm{C}_{3} \mathrm{~N}_{4}$ Sheet-on-sheet Nanocomposites for Efficient Visible-light Photocatalytic $\mathrm{H}_{2}$-evolution and Degradation of Organic Pollutants. RSC 
Advances, 5, 97951-97961.

[38] Zhang, S., Yang, Y., Guo, Y. (2013) Preparation and Enhanced Visible-light Photocatalytic Activity of Graphitic Carbon Nitride/Bismuth Niobate Heterojunctions. J Hazard Mater, 261, 235-245.

[39] Chang, C., Fu, Y., Hu, M. (2013) Photodegradation of Bisphenol A by Highly Stable Palladium-doped Mesoporous Graphite Carbon Nitride $\left(\mathrm{Pd} / \mathrm{mpg}^{-} \mathrm{C}_{3} \mathrm{~N}_{4}\right)$ under Simulated Solar Light Irradiation. Applied Catalysis B: Environmental, 142-143.

[40] Cao J., Zhao, Y., Lin, H. (2013) Ag/AgBr/g-C $\mathrm{N}_{4}$ : A Highly Efficient and Stable Composite Photocatalyst for Degradation of Organic Contaminants under Visible Light. Materials Research Bulletin, 48, 3873-3880. 